\title{
The Polycarboxylate-based Superplasticizers Modified with thickening agents: the performance and Influece on Cement Hydration
}

\author{
Jing Feng ${ }^{1,2,3, \mathrm{a}^{*}}$, Jiaxiang $\mathrm{Li}^{3, \mathrm{~b}}$, Liang Chen ${ }^{1,2,3, \mathrm{c}}$, Pengxiang $\mathrm{Li}^{1, \mathrm{~d}}$, and Zaiqin \\ Wang ${ }^{1,2,3, \mathrm{e}}$ \\ ${ }^{1}$ Changjiang River Scientific Research Institute, Wuhan, 430010, China \\ ${ }^{2}$ National Dam Safety Research Center,Wuhan, 430010, China \\ ${ }^{3}$ Collaborative Innovation Center for Geo-hazards and Eco-Environment in Three Gorges \\ Area, Yichang, 443002, China \\ a980806fj@163.com, b173605993@qq.com, cchenliang@mail.crsri.cn, dlipx@mail.crsri.cn, \\ ewangzq@mail.crsri.cn
}

\begin{abstract}
Keywords: Polycarboxylate-based superplasticizer, thickening agent, water-reducing ratio, compressive strength, cement hydration.

Abstract. The application of effective superplasticizer is helpful to improve the working performance and durability of concrete. In our work, several high performance polycarboxylate-based superplasticizers were prepared by modifying the commercial products with two types of thickening agents. Their dispersion ability for cement was investigated by fluidity and bleeding tests, while their influences on mortar working performance were evaluated based on the water-reducing ratio and the compressive strength experiments. The hydrated products of cement mortar were characterized by SEM and DSC-TGA technologies as well. Results indicated that three types of superplasticizers, including PCA, PCA-1 and PCA-2, exhibited good cement compatibility and water-reducing effects. These superplasticizers delayed the initial period of cement hydration, and made the hydration products of mortar develop into the more compact and ordered structures, consequently leading to the higher compressive strength.
\end{abstract}

\section{Introduction}

Polycarboxylate-type superplasticizer (PCE) is one of the indispensable materials for high-fluidity and high-strength concrete because of its superior dispersing force for cement, especially at low water/cement ratios, and its retention effects. However, it has been known that the dispersing force of PCE for cement and its time variation are significantly affected by even slight differences in concrete components or mixing conditions. For example, it often leads to bleeding when the dosage of PCE is slightly higher than the medium level. Such instability of the dispersing force is explained by the incompatibility between cement and $\mathrm{PCE}^{[1-3]}$. The application of effective thickening agents is helpful to improve their compatibility, and subsequently increase the cohesiveness and durability of concrete. Herein, two types of thickener were used respectively to modify the commercial polycarboxylate products, thus obtained superplasticizers were evaluated about their water-reducing effect and workability. Their effects on the compressive strength and hydration products of mortar were discussed as well. This work is beneficial for the development of high performance superplasticizers wiht good cement compatibility.

\section{Experimental}

\section{Materials}

Two kinds of commercial polycarboxylate-type superplasticizers, namely PC and PCA, were used. Their main component was co-polymer of methacrylate containing poly(ethyleneoxide) (PEO) graft chain. The organic thickener Z1 was used to modify the PC and PCA, and thus obtained the products PC-1 and PCA-1, respectively. The inorganic thickener Z2 was also used to modify the PC and PCA, and thus obtained the products PC-2 and PCA-2, respectively. The cement used was a commercial 
normal Portland cement.

\section{Methods}

Fluidity tests for cement paste: Cement pastes were mixed at $20^{\circ} \mathrm{C}$ and at $\mathrm{w} / \mathrm{c}$ of 0.35 . The amount of superplasticizer added is expressed as a percentage of dry solid with respect to the mass of cement. The fluidity of cement paste was evaluated by a flow test. Flow was measured at $20^{\circ} \mathrm{C}$ by pullout spread of cement paste from a pipe of 50-mm inner diameter and 51-mm height.

Bleeding ratio tests for cement paste: According to the fluidity test method for cement paste, the cement paste at $\mathrm{w} / \mathrm{c}$ of 0.35 was covered and standing until no bleeding was observed. During the first one hour, removed bleeding water at an interval of $10 \mathrm{~min}$. After while, repeated it an interval of 30 min. Bleeding ratio $(\%)=$ the mass of bleeding water $/$ the total mass of water $\times 100 \%$.

Working performance of cement mortar : According to the concrete admixture homogeneity test method(see GB/T 8077), the water-reducing ratio of mortar was tested as follows. Firstly, the cement $(450 \mathrm{~g})$ and standard sand $(1350 \pm 5 \mathrm{~g})$ were mixed with water, then record the amount of water when the mortar fluidity reaches $(180 \pm 5) \mathrm{mm}$. Water-reducing ratio is calculated by measuring the variation of water amount after adding different kinds of polycarboxylate products. According to the cement mortar strength test method (see GB/T 17671), the compressive strength of motar at the age of $3 \mathrm{~d}, 7 \mathrm{~d}$ and $28 \mathrm{~d}$ were tested respectivle. In all aboved experiments, the mortar without any superplasticizers (marked as "Blank") were used as references.

Characterization of cement mortar hydration products: The DSC-TGA test was used to evaluated the content of calcium hydroxide $(\mathrm{CH})$ in motar hydration products at the age of $3 \mathrm{~d}, 7 \mathrm{~d}$ and $28 \mathrm{~d}$ respectively. The SEM technology was used to characterize the morphology of motar hydration products. In all aboved experiments, the mortar without any superplasticizers (marked as "Blank") were used as references.

\section{Results and Discussion}

The fluidity and bleeding ratio of fresh cement paste

Table 1 Fluidity and Bleeding ratio of fresh cement paste

\begin{tabular}{ccccc}
\hline No. & $\begin{array}{c}\text { Type of } \\
\text { superplasticizer }\end{array}$ & $\begin{array}{c}\text { Amount of } \\
\text { superplasticizer } \\
(\mathrm{wt} \%)\end{array}$ & $\begin{array}{c}\text { Fluidity } \\
(\mathrm{mm})\end{array}$ & Bleeding ratio (\%) \\
\hline 1 & PC & 0.15 & 137 & 0.0 \\
2 & PC & 0.20 & 180 & 0.0 \\
3 & PC & 0.30 & 223 & 3.1 \\
4 & PCA & 0.15 & 206 & 0.0 \\
5 & PCA & 0.20 & 225 & 2.4 \\
6 & PC1 & 0.30 & 222 & 0.8 \\
7 & PC2 & 0.30 & 197 & 0.4 \\
8 & PCA-1 & 0.20 & 232 & 0.1 \\
9 & PCA-2 & 0.20 & 221 & 0.1 \\
\hline
\end{tabular}

Table 1 shows the fluidity and bleeding results of fresh cement paste in the presence of several different polycarboxylate superplasticizers. As shown in table 1, the bleeding phenomenon appeared when the amount of commercial polycarboxylate superplasticizer PC increased to $0.3 \%$, while that of PCA increased to $0.2 \%$. However, when the modified superplasticizers (including PC-1, PC-2, PCA-1 and PCA-2) were added to the cement respectively, bleeding phenomenon delayed and bleeding ratio decreased significantly (Table 1). Such results indicated that both thickening agents (Z1 and Z2) improved the cement dispersion effects and compatibility of the polycarboxylate superplasticizer. 


\section{Working performance of cement mortar}

As shown in Table 2, the mortar added with PCA and its modified products ( PCA-1 and PCA-2 ) exhibited relatively higher water-reducing ratio, and showed no bleeding phenomenon. Combined with the cement paste fluidity and bleeding results (Table 1), it suggested that the PCA and its modified products exhibited better cement dispersion ability and compatibility.

Table 2 Water-reducing ratio of mortar added with $0.2 \%$ of superplasticizers

\begin{tabular}{ccc}
\hline No. & Type of superplasticizer & $\begin{array}{c}\text { Water-reducing ratio } \\
(\%)\end{array}$ \\
\hline 1 & PC & 16.12 \\
2 & PC-1 & 15.32 \\
3 & PC-2 & 15.09 \\
4 & PCA & 19.66 \\
5 & PCA-1 & 20.21 \\
6 & PCA-2 & 19.80 \\
\hline
\end{tabular}

The compressive strength of the mortar added with PCA, PCA-1 and PCA-2, respectively, were shown in Figure 1. Compared with the cement mortar without adding any superplasticizer (Blank), the compressive strength of mortar added with PCA, PCA-1 and PCA- 2 at the age of $3 \mathrm{~d}$ increased by $67.4 \% \sim 91.1 \%$, the strength at the age of $7 \mathrm{~d}$ increased by $19.3 \% \sim 36.6 \%$, and the strength at the age of $28 \mathrm{~d}$ increased by $7.0 \% \sim 24.1 \%$. Such results may related to the differences in their mortar hydration products.

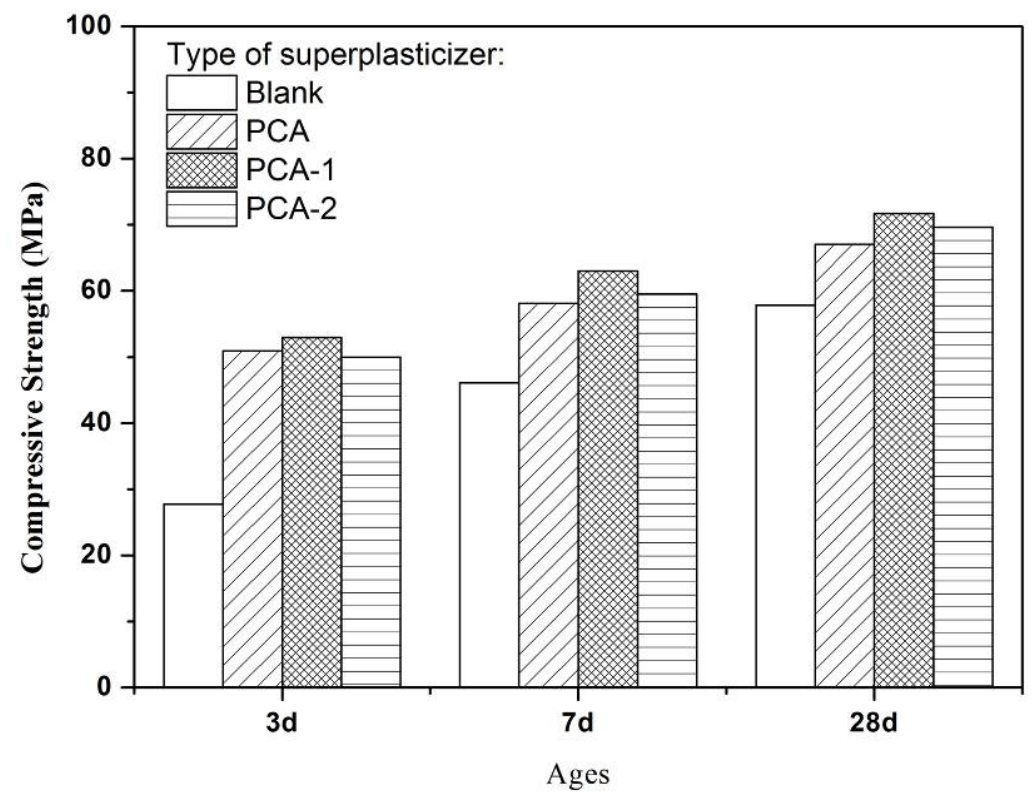

Fig. 1 The compressive strength of mortar at different ages

\section{Effects of superplasticizers on cement hydration process}

There are mainly four types of cement hydration products, including calcium silicate hydrate $(\mathrm{C}-\mathrm{S}-\mathrm{H})$ gel, calcium hydroxide $(\mathrm{CH})$, ettringite $(\mathrm{Aft})$ and calcium monosulfate aluminate hydrate (AFm). Usually the hydration degree is proportional to the content of calcium hydroxide $(\mathrm{CH})$ in hydration products. And the thermal weight loss ranged from $400{ }^{\circ} \mathrm{C}$ to $550{ }^{\circ} \mathrm{C}$ in $\mathrm{TG}$ curve often suggests the decomposition of $\mathrm{CH}$. Hence, the hydration degree of cement mortar could be deduced by calculating the $\mathrm{CH}$ content in the hydration products using DSC-TGA test ${ }^{[4]}$. As shown in Figure 2 , the $\mathrm{CH}$ contents of mortar with three different water-reducing agents (PCA,PCA-1 and PCA-2) at different ages were all lower than that of the blank mortar, especially those at $3 \mathrm{~d}$ ages. Such results 
suggested that these three kinds of water-reducing agent probably delayed the early hydration process.

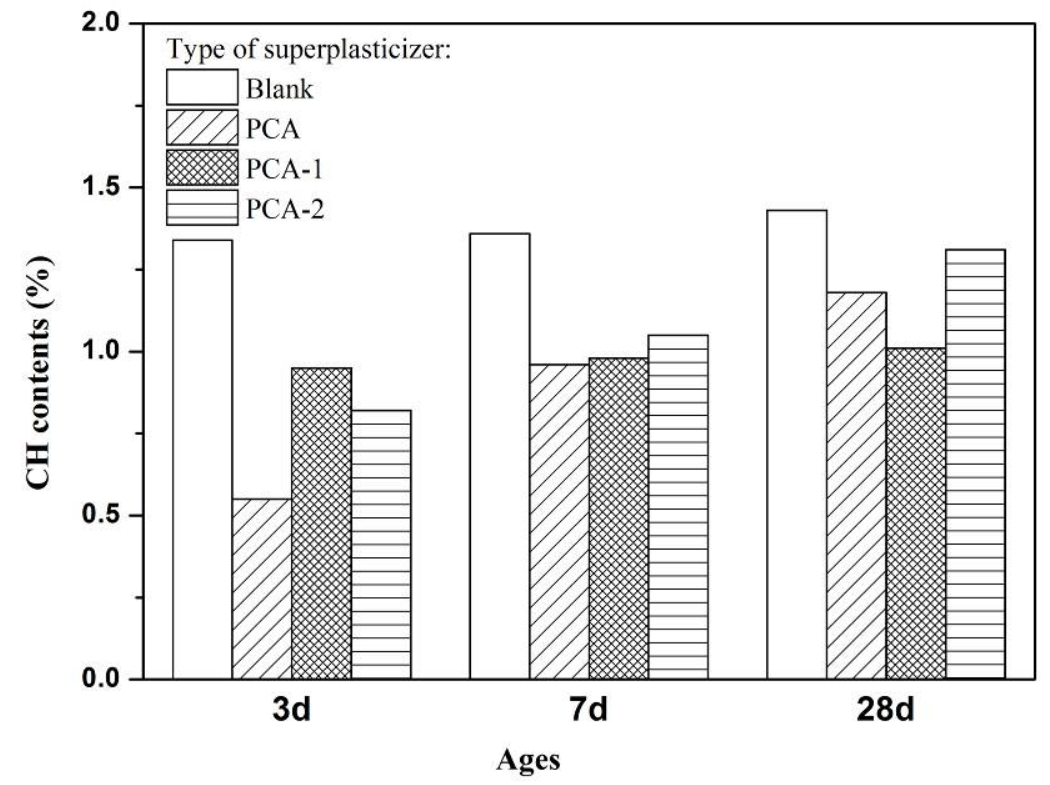

Fig. 2 The $\mathrm{CH}$ content in the mortar hydration products at different ages
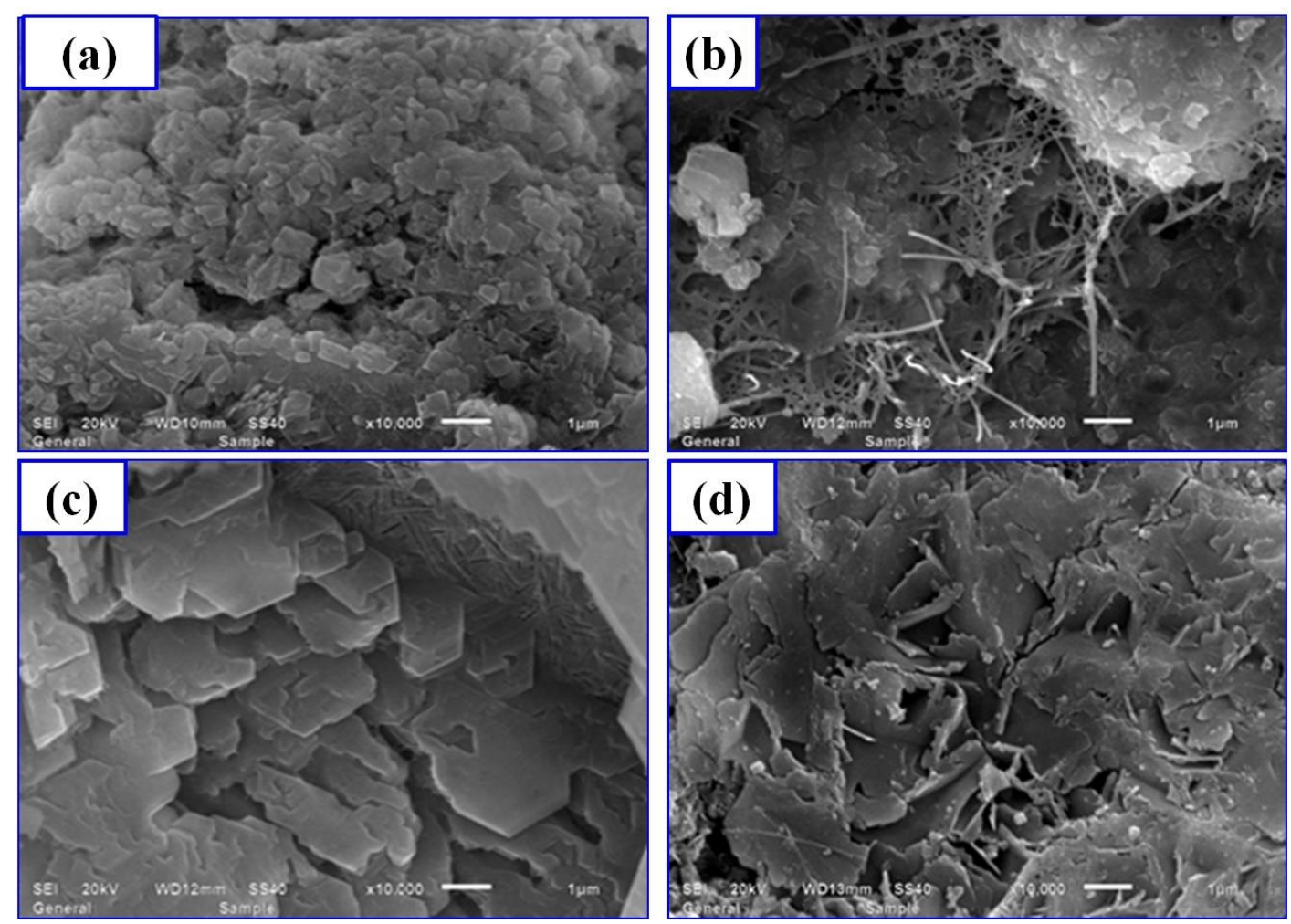

Fig.3 Hydration products morphology of the mortar added with the superplasticizer: a) none (Blank); b) PCA; c) PCA-1; d)PCA-2.

The morphology of mortar hydration products at the age of $28 \mathrm{~d}$ were characterized by SEM. As shown in Figure 3, all of these three water-reducing agents (PCA,PCA-1 and PCA-2) were helpful to the development of the hydration products, promoting the calcium silicate hydrate $(\mathrm{C}-\mathrm{S}-\mathrm{H})$ gel to develop into the more compact and ordered crystal structures. In combination with the aforementioned compressive strength and $\mathrm{CH}$ contents results, it also suggested that these 
water-reducing agents improved the strength and structure compactness of mortar, which was in consistence with the previous reports ${ }^{[5,6]}$.

\section{Conclusions}

In our work, two types of commercial polycarboxylate-based superplasticizers (PC and PCA) and their modification products (PC-1,PC-2, PCA-1 and PCA-2) were respectively added to the Portland cement paste and mortar. Results showed that the modification of the commercial products by thickening agents is helpful to improve their dispersion ability and compatibility for cement. Among all those superplasticizers, the PCA, PCA-1 and PCA- 2 contributed to the higher water-reducing ratio and compressive strength of cement mortar. And as confirmed by the results of thermal analysis and SEM tests, their better working performance was related to their influences on cement hydration products. The superplasticizer PCA, PCA-1 and PCA-2 delayed the initial period of cement hydration,made the hydration products develop into a more compact and ordered structure, and then lead to the higher compressive strength.

\section{Acknowledgements}

This work was financially supported by National Natural Science Foundation of China (51309018), Non-Profit Industry Financial Program of MWR (Grant No. 201301023), the Fundamental Research Funds for the Central Research Institute (CKSF 2015032/CL), and by the International Science \& Technology Cooperation Program of China (Grant No. 2010DFB70470). Thanks for the supporting by Innovation Team of Changjiang River Scientific Research Institute.

\section{References}

[1] S. Hanehara, K. Yamada. Interaction between cement and chemical admixture from the points of cement hydration, admixture adsorption and paste rheology [J]. Cem. Concr. Res., 1999, 29 (8): 1159- 1165.

[2] P.C. Aictin, C. Jolicoeur, J.G. MacGregor. Superplasticizers: how they work and why they occasionally don't [J]. Concr. Int., 1994, 5: 45-52.

[3] A. Tagnit-Hamou, P.C. Aitcin. Cement and superplasticizer compatibility [J]. World Cem., 1993, 24 (8): 38-42. [4] Peiming Wang, Shuxia Feng, Xianping Liu. Research approaches of cement hydration degree and their development [J]. J. Build. Mater., 2005, 8(6): 646-652. (In Chinese)

[5] Xuehong Huang, Pu Lin, Muxia Zheng, etc. Influence of the polycarboxylate on cement hydration [J]. Guangzhou Chem., 2008, 33(1) : 14-19. (In Chinese)

[6] Shenghua Lv, Chaochao Qiu, Ruijun Gao, etc. Effect of $\beta$-cyclodextrin side groups on structure and properties of new polycarboxylate superplasticizer [J]. Func. Mater., 2013,44(1) : 56-60. (In Chinese) 